\section{(2) OPEN ACCESS}

\title{
Cost-effectiveness of transcatheter edge-to-edge repair in secondary mitral regurgitation
}

\author{
David J Cohen, ${ }^{1,2}$ Kaijun Wang, ${ }^{3}$ Elizabeth Magnuson, ${ }^{3}$ Robert Smith, ${ }^{4}$ \\ Mark C Petrie, ${ }^{5,6}$ Mamta Heena Buch, ${ }^{7}$ William Abraham ${ }^{8}$ Joann Lindenfeld, ${ }^{9}$ \\ Michael J Mack, ${ }^{10}$ Gregg W Stone, ${ }^{11,12}$ John G F Cleland ${ }^{13,14}$
}

- Additional supplemental material is published online only. To view, please visit the journal online (http://dx.doi. org/10.1136/heartjnl-2021320005).

For numbered affiliations see end of article.

\section{Correspondence to}

Dr David J Cohen, Clinical Trials Center, Cardiovascular Research Foundation, New York, NY 10019, USA; dcohen@crf.org

Received 13 September 2021 Accepted 28 December 2021

Check for updates

(C) Author(s) (or their employer(s)) 2022. Re-use permitted under CC BY-NC. No commercial re-use. See rights and permissions. Published by BMJ.

To cite: Cohen DJ, Wang K, Magnuson E, et al. Heart Epub ahead of print: [please include Day Month Year]. doi:10.1136/ heartjnl-2021-320005

\begin{abstract}
Background Transcatheter edge-to-edge mitral valve repair (TMVr) improves symptoms and survival for patients with heart failure with reduced left ventricular ejection fraction (HFrEF) and severe secondary mitral regurgitation despite guideline-recommended medical therapy (GRMT). Whether TMVr is cost-effective from a UK National Health Service (NHS) perspective is unknown.

Methods We used patient-level data from the Cardiovascular Outcomes Assessment of the MitraClip Percutaneous Therapy for Heart Failure Patients with Functional Mitral Regurgitation (COAPT) trial to perform a cost-effectiveness analysis of TMVr +GRMT versus GRMT alone from an NHS perspective. Costs for the TMVr procedure were based on standard English tariffs and device costs. Subsequent costs were estimated based on data acquired during the trial. Health utilities were estimated using the Short-Form 6-Dimension Health Survey.
\end{abstract}

Results Costs for the index procedural hospitalisation

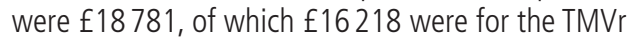
device. Over 2-year follow-up, TMVr reduced subsequent costs compared with GRMT ( $\mathrm{f} 10944$ vs $\mathrm{f} 14932$, $p=0.006$ ), driven mainly by reductions in heart failure hospitalisations; nonetheless, total 2-year costs remained higher with TMVr (£29 165 vs $£ 14932, p<0.001$ ). When survival, health utilities and costs were projected over a lifetime, TMVr was projected to increase life expectancy by 1.57 years and quality-adjusted life expectancy by 1.12 quality-adjusted life-years (QALYS) at an incremental cost of $£ 21980$, resulting in an incremental cost-effectiveness ratio (ICER) of $£ 23270$ per QALY gained (after discounting). If the benefits of TMVr observed in the first 2 years were maintained without attenuation, the ICER improved to $\mathrm{f} 12494$ per QALY. Conclusions For patients with HFrEF and severe secondary mitral regurgitation similar to those enrolled in COAPT, TMVr increases life expectancy and qualityadjusted life expectancy compared with GRMT at an ICER that represents good value from an NHS perspective.

For patients with heart failure with reduced ejection fraction (HFrEF) and severe secondary mitral regurgitation (MR), the Cardiovascular Outcomes Assessment of the MitraClip Percutaneous Therapy for Heart Failure Patients with Functional Mitral Regurgitation (COAPT) trial showed that edgeto-edge transcatheter mitral valve repair (TMVr), when added to guideline-recommended medical therapy (GRMT), ${ }^{12}$ improved quality of life (QoL) and reduced hospitalisations for heart failure (HHF) and mortality. A cost-effectiveness analysis from a US healthcare perspective found that the incremental cost-effectiveness ratio (ICER) for TMVr was $\sim \$ 55000$ per quality-adjusted lifeyears (QALY) gained compared with GRMT, which is considered reasonably cost-effective from a US perspective. $^{3}{ }^{4}$ However, there is little information regarding the cost-effectiveness of $\mathrm{TMVr}$ in other healthcare systems that may differ in terms of resource utilisation patterns, costs, population life expectancy and values assigned to health states. To address this gap in knowledge, we performed a cost-effectiveness analysis of TMVr from a UK National Health Service (NHS) perspective.

\section{METHODS \\ Design and Patient population}

This study was based on individual-patient data from the COAPT trial and is reported according to Consolidated Health Economic Evaluation Reporting Standards (CHEERS) guidelines. ${ }^{5}$ COAPT (clinicaltrials.gov identifier NCT 01626079) enrolled patients with symptomatic heart failure, left ventricular ejection fraction (LVEF) 20\%-50\% and severe $(3+$ or $4+)$ secondary MR. After optimisation of GRMT, patients were randomised to receive either TMVr using the MitraClip device + GRMT (hereafter referred to as TMVr) or GRMT alone. Patients were followed up for 2 years, after which patients assigned to GRMT were allowed to undergo TMVr.

\section{Ethics}

The trial was approved by relevant institutional review boards, and informed consent was obtained from all patients. The economic analysis was approved by the institutional review board at Saint Luke's Hospital of Kansas City.

\section{Analytic overview}

The study design was similar to the US costeffectiveness analysis, ${ }^{3}$ included all randomised patients, and was analysed according to intentionto-treat. Detailed medical resource utilisation, vital status and QoL were recorded using standardised case report forms from randomisation through 2-year follow-up. Observed within-trial data were then used to project patient-level survival, health 
utilities and costs over a lifetime perspective. Cost-effectiveness was calculated both as cost per QALY gained and cost per lifeyear gained (LYG).

\section{Within-trial costs}

Within-trial costs were assessed in 2019 pounds sterling $(£)$ using measured resource utilisation and unit costs appropriate to the UK NHS. For the initial TMVr procedure and hospitalisation, costs were assigned based on standard English tariffs for elective and urgent admissions ( $£ 2514$ and $£ 4869$, respectively) and the current cost of the TMVr device ( $£ 16500$ per procedure, regardless of the number of devices used). Costs for additional resources and physician services during the index hospitalisation were not assessed because these costs are included in the tariff. For each subsequent hospitalisation, principal diagnosis, major procedures and admission status (elective or unplanned) were recorded. Costs were then calculated using NHS National Payment by Results (PbR) tariffs. ${ }^{6}$ Costs for emergency department visits were assigned based on the tariff for Emergency Medicine (Category 3 investigation with Category 1-3 treatment). Medication costs were based on the British National Formulary. ${ }^{7}$ Costs associated with inpatient rehabilitation services and skilled-nursing facility stays were assigned based on mean length of stay multiplied by NHS reference costs. ${ }^{8}$

\section{Estimates of life-years gained and quality-adjusted life-years} Projected survival beyond 2 years was estimated separately for the TMVr and GRMT groups. For the GRMT group, 2-year survival was compared with expected age-adjusted and sexadjusted mortality using UK life-tables ${ }^{9}$ to calculate a calibration factor (relative mortality hazard). For each 2-year survivor, life expectancy beyond 2 years (or the last observed follow-up) was then estimated from recalibrated life-tables. ${ }^{31011}$ Long-term survival for 2-year survivors in the TMVr group was estimated in a similar fashion after applying the HR for mortality after TMVr versus GRMT based on landmark analysis of trial data between 30 days and 2 years.

QoL was assessed at baseline and at 1, 6, 12 and 24 months using the Short-Form 36 health-status instrument (SF-36). Individual responses were converted to utility weights for the UK population, ${ }^{12}$ and QALYs were calculated as the time-weighted average of utility values. Utilities after the within-trial period were estimated from a linear regression model adjusted for age, sex, baseline utility, treatment group, stroke and left ventricular assist device (LVAD) or cardiac transplantation. QALYs beyond the in-trial period were calculated by multiplying estimated survival (within 30-day intervals) by predicted utilities.

Similar to utilities, future healthcare costs were estimated on the basis of a linear regression model (which included age, sex and treatment group as covariates) derived from observed costs in the second year after randomisation.

\section{Statistical analysis}

Continuous data are reported as mean \pm SD or median with 25 th and 75 th percentiles and compared by t-tests or Wilcoxon rank-sum tests as appropriate. Categorical data are reported as frequencies and compared by Fisher's exact test. Cost data are reported as both mean and median values and compared using non-parametric bootstrapping (1000 replicates). Time-to-event data are reported as 2-year Kaplan-Meier estimates and were compared using a Cox proportional hazards model. To account for patient dropout, methods for the analysis of censored data were used to estimate costs, resource use and utilities at each follow-up timepoint, ${ }^{13}$ and bootstrapping was used to calculate confidence intervals associated with these estimates. ${ }^{14}$ Group means and between-group differences for projected LYG, QALYs and lifetime costs were generated with bootstrap resampling. All analyses were performed using SAS V.9.4 (SAS Institute). For all comparisons, a $\mathrm{p}$ value $<0.05$ was considered statistically significant. By contractual arrangement with the study sponsor, patient-level data will not be shared.

\section{Cost-effectiveness analysis}

For the purposes of cost-effectiveness analyses, all future costs and health benefits were discounted at $3.5 \%$ per year. ${ }^{15}$ ICERs were calculated as the difference in discounted lifetime costs divided by the difference in discounted LYG or QALYs. Uncertainty in the joint distribution of these differences and the resulting ICERs was estimated using bootstrap resampling.

Since the duration of the survival, QoL and follow-up cost benefits of TMVr beyond the 2-year trial period are unknown, three scenarios were considered. The base-case analysis assumed that the benefits of TMVr decreased in a linear fashion from year 2 to year 5, such that there was no further benefit of TMVr beyond year 5 . Two alternative scenarios were also modelled under the assumptions that the benefits of TMVr observed at the end of the trial period remained constant throughout the patient's lifetime (best-case scenario) or that the in-trial benefits of TMVr did not extend beyond 2 years (worst-case scenario).

To account for differences in practice between the USA (where the trial was conducted) and the UK, we also performed a sensitivity analysis in which patients who underwent LVAD placement or cardiac transplantation at age $>65$ years were assumed to have died rather than receiving these interventions. Because some guidelines recommend that cost-effectiveness analyses exclude unrelated costs in future years, we performed a sensitivity analysis using this approach. Finally, lifetime costeffectiveness was estimated for subgroups according to sex, age, Society of Thoracic Surgeons Mortality Risk score, severity of tricuspid regurgitation, LVEF, type of cardiomyopathy (ischaemic vs non-ischaemic), severity of MR and symptom severity.

\section{Patient involvement}

Patients were not directly involved in the design or conduct of the COAPT trial or the associated economic analysis.

\section{RESULTS}

\section{Patient population}

Altogether, 614 patients were enrolled in COAPT and randomised to either TMVr + GRMT $(n=302)$ or GRMT alone $(n=312)$. Baseline characteristics were well matched (online supplemental table A). Median age was 74 years, most patients were male, and most patients had multiple comorbidities.

\section{Index hospitalisation costs}

Of the 302 patients assigned to TMVr, implantation was attempted in 293, with a median procedure duration of $171 \mathrm{~min}$ (table 1). The mean cost of the index hospitalisation was $£ 18781$ (median $£ 19014$ ), of which $£ 16218$ (median $£ 16218$ ) was related to the TMVr device, itself, with the remaining $£ 2562$ (median $£ 2514$ ) reflecting the tariff for the procedure and hospitalisation.

\section{Follow-up clinical outcomes, resource use and costs}

At 2 years, all-cause mortality (28.2\% vs $43.0 \%)$, HHF $(34.8 \%$ vs $56.4 \%$ ) and the composite of death or HHF (44.8\% vs $67.0 \%)$ 
Table 1 Index hospitalisation resource use and costs for patients who underwent attempted TMVr

\begin{tabular}{ll}
\hline Resource category & $\begin{array}{l}\text { TMVr } \\
(\mathbf{n}=293)\end{array}$ \\
\hline Procedure duration (min) & $171 \pm 110$ \\
\hline Length of stay (days) & $0.6 \pm 1.2(0)$ \\
\hline ICU & $1.9 \pm 2.0(1)$ \\
\hline Non-ICU & $2.5 \pm 2.3(2)$ \\
\hline Total & \\
\hline Index hospitalisation events & $4(1.4 \%)$ \\
\hline Death & $1(0.3 \%)$ \\
\hline MI & $1(0.3 \%)$ \\
\hline Stroke & $0(0 \%)$ \\
\hline Repeat mitral valve procedure & \\
\hline Vascular complication & \\
\hline Index hospitalisation costs (f) & $16218 \pm 2141$ (16500) \\
\hline MitraClip devices & $2562 \pm 334(2514)$ \\
\hline Hospital tariff & $18781 \pm 2173(19014)$ \\
\hline Total index admission costs & \\
\hline Values are mean $\pm S D$ (median). \\
ICU, intensive care unit; MI, myocardial infarction ; TMVr, transcatheter edge-to-edge mitral \\
valve repair.
\end{tabular}

were lower with TMVr compared with GRMT alone (table 2; all $\mathrm{p}<0.001)$. The rates of other clinical endpoints including stroke, myocardial infarction (MI) or mitral valve (re-)intervention were similar between groups. Hospitalisations (per 100 patients) were reduced from 217 in the GRMT group to 169 in the TMVr group, driven largely by a reduction in HHF (table 2). There were also fewer other cardiovascular and non-cardiovascular hospitalisations, emergency department visits and HF-related office visits in the TMVr group. As a result, follow-up costs were reduced by $£ 3988$ per patient with TMVr compared with GRMT (95\%CI for difference, $£ 1257$ to $£ 6933$; $\mathrm{p}=0.006$ ). However, when combined with the costs of TMVr, total 2-year costs for the TMVr group were higher than for GRMT alone ( $£ 29165$ vs $£ 14932$; mean difference $£ 14233$ (95\% CI $£ 11324$ to $£ 16910)$; $\mathrm{p}<0.001)$.

\section{In-trial utilities and QALYs}

Mean in-trial survival duration was greater with TMVr than with GRMT (1.62 years vs 1.46 years) as were utility scores (online supplemental table B). As a result, in-trial QALYs were 1.15 years vs 1.00 years for TMVr and GRMT, respectively (mean difference 0.14 QALYs (95\% CI 0.07 to 0.21 ); $\mathrm{p}<0.001$ ).

\section{Lifetime projections}

Under our base-case assumptions (which assumed gradual loss of benefit after 2 years), undiscounted life expectancy was projected to be 6.56 years with TMVr and 4.98 years with GRMT (mean difference 1.57 years, 95\% CI 0.62 to 2.59 ; figure 1). Qualityadjusted life expectancy was projected to be 4.31 and 3.19 QALYs with TMVr and GRMT, respectively (mean difference 1.12 QALYs, 95\% CI 0.48 to 1.80). Finally, discounted lifetime medical costs were projected to be $£ 59970$ and $£ 37990$ for the TMVr and GRMT groups, a difference of $£ 21980$ (95\% CI $£ 14760$ to $£ 29248)$.

\section{Cost-effectiveness analyses}

Figure 2A shows the joint distributions of the projected differences in discounted lifetime costs and QALYs based on bootstrap replication. Based on these projections, the ICER for TMVr vs

\begin{tabular}{|c|c|c|c|c|}
\hline & $\begin{array}{l}\text { TMVr } \\
\mathrm{n}=302\end{array}$ & $\begin{array}{l}\text { GRMT } \\
n=312\end{array}$ & $\begin{array}{l}\text { HR or } \\
\text { difference }(95 \% \mathrm{Cl})\end{array}$ & $P$ value \\
\hline \multicolumn{5}{|l|}{ 2-Year clinical outcomes* } \\
\hline Death (n, \%) & $83(28.2)$ & $125(43.0)$ & 0.62 (0.47 to 0.82$)$ & $<0.001$ \\
\hline Stroke $(\mathrm{n}, \%)$ & $11(4.2)$ & $15(6.5)$ & $0.70(0.32$ to 1.52$)$ & 0.367 \\
\hline $\mathrm{MI}(\mathrm{n}, \%)$ & $0(0)$ & $0(0)$ & 0 (NA) & NA \\
\hline Repeat valve intervention (n, \%) & $1(0.4)$ & $6(2.1)$ & $0.17(0.02$ to 1.40$)$ & 0.099 \\
\hline Hospitalisations (any) $†$ & 169 (147 to 191$)$ & 217 (195 to 241) & $-48(-84$ to -16$)$ & 0.004 \\
\hline Heart failuret & 57 (44 to 70$)$ & 96 (82 to 111$)$ & $-38(-60$ to -18$)$ & $<0.001$ \\
\hline Cardiovascular but not heart failuret & 33 (26 to 41$)$ & 37 (30 to 45$)$ & $-4(-14$ to 6$)$ & 0.528 \\
\hline Non-cardiovasculart & 79 (66 to 92$)$ & 85 (71 to 100$)$ & $-6(-27$ to 13$)$ & 0.52 \\
\hline Hospital days $†$ & 1050 (835 to 1283) & 1372 (1166 to 1616$)$ & $-322(-653$ to 5$)$ & 0.056 \\
\hline SNF/rehab days $t$ & 366 (311 to 423) & 471 (409 to 537) & $-105(-194$ to -15$)$ & 0.02 \\
\hline Emergency room visitst & 52 (41 to 65$)$ & 56 (43 to 70$)$ & $-4(-20$ to 13$)$ & 0.684 \\
\hline Heart failure-related office visitst & 98 (70 to 132$)$ & 119 (87 to 155$)$ & $-20(-67$ to 30$)$ & 0.4 \\
\hline \multicolumn{5}{|l|}{ Costs (f) } \\
\hline Hospitalisations & 8213 (6579 to 9988) & 11935 (10065 to 14080$)$ & $-3722(-6477$ to -1106$)$ & 0.004 \\
\hline Outpatient services & 2730 (2466 to 2996$)$ & 2996 (2646 to 3358) & $-266(-707$ to 185$)$ & 0.252 \\
\hline SNF/rehab services & 436 (371 to 504$)$ & 561 (488 to 641) & $-125(-232$ to -18$)$ & 0.02 \\
\hline Medications & 2012 (1770 to 2265) & 2111 (1786 to 2460$)$ & $-99(-507$ to 320$)$ & 0.672 \\
\hline ED visits & 130 (104 to 163$)$ & 140 (109 to 176$)$ & $-10(-50$ to 32$)$ & 0.684 \\
\hline Heart failure-related office visits & 152 (108 to 205) & 184 (135 to 240$)$ & $-32(-104$ to 46$)$ & 0.4 \\
\hline Total follow-up costs (f) & 10944 (9254 to 12775$)$ & 14932 (12981 to 17027$)$ & $-3988(-6933$ to -1257$)$ & 0.006 \\
\hline Cumulative 2-year costs (f) & 29165 (27541 to 31054$)$ & 14932 (12981 to 17027$)$ & 14233 (11324 to 16910$)$ & $<0.001$ \\
\hline
\end{tabular}




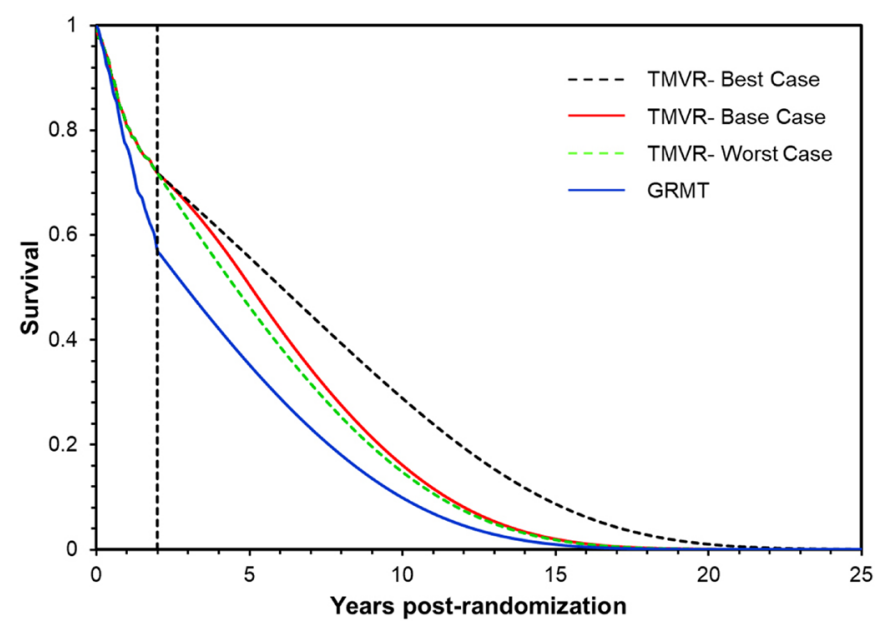

Figure 1 Survival projections for transcatheter mitral valve repair (TMVr) and guideline-recommended medical therapy (GRMT). Survival probability projections based on 2-year observed outcomes and recalibrated life-tables for TMVr base case (red), TMVr best case (purple dash), TMVr worst case (green dash) and GRMT (blue). See the Methods section for details of projections and scenarios.

GRMT was $£ 23270$ per QALY gained. The probability that TMVr would provide high economic value (ie, ICER $<£ 20000$ per QALY gained) was $18 \%$, while the probability that TMVr would provide good economic value (ICER $<£ 30000$ per QALY gained) was $89 \%$ (figure 3 ). When benefits were assessed in lifeyears rather than QALYs, the ICER was $£ 17140$ per LYG, and the probabilities that the ICER was $<£ 20000$ or $<£ 30000$ per LYG were $76 \%$ and $96 \%$, respectively (figures $2 \mathrm{~B}$ and 3 ).

\section{Sensitivity and subgroup analyses}

Varying assumptions regarding the duration of benefit of TMVr resulted in modest alterations in estimated ICERs (table 3). Under the best-case scenario, TMVr was associated with an ICER of $£ 12494$ per QALY, while the ICER increased to $£ 28607$ per QALY under our worst-case scenario-differences that were driven mainly by projected changes in life

A

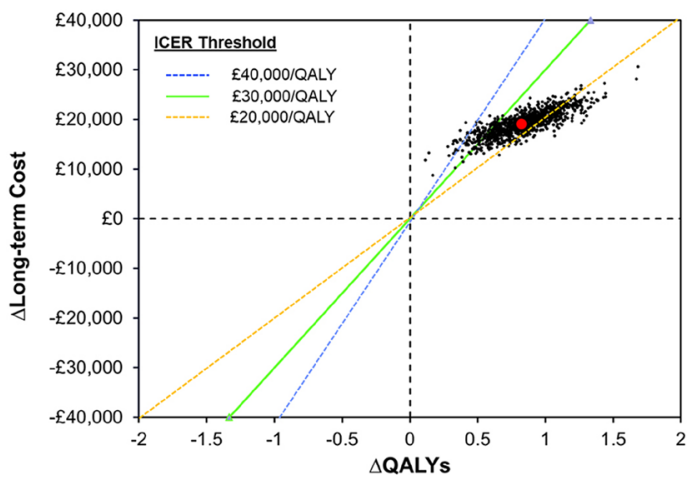

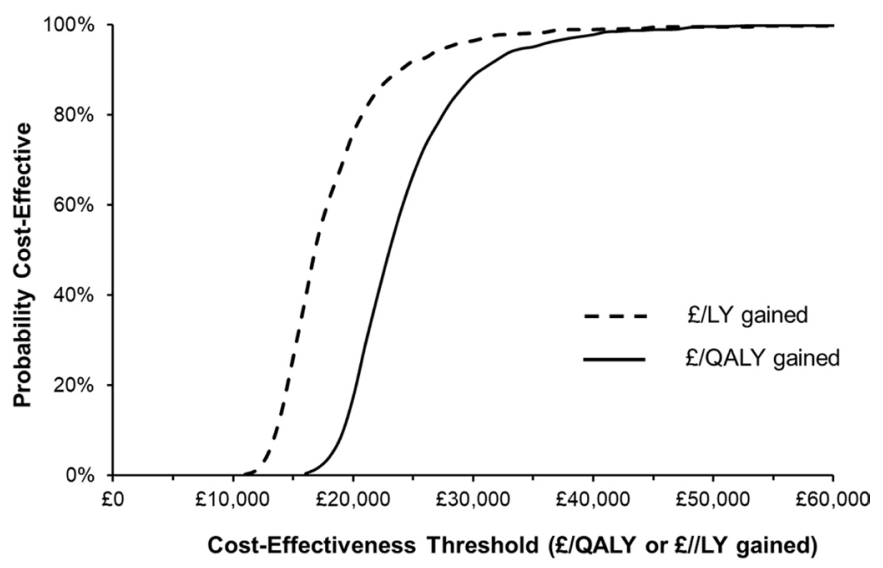

Figure 3 Cost-effectiveness acceptability curves for transcatheter mitral valve repair versus guideline recommended medical therapy. The graph displays the probability that TMVr is cost-effective, calculated as the proportion of bootstrap iterations that fall below a given cost-effectiveness threshold, plotted across a range of possible costeffectiveness thresholds expressed as both $\mathrm{f}$ per QALY gained and $\mathrm{f}$ per LY gained. LY, lifeyear; QALY, quality-adjusted life-year.

expectancy gains with TMVr. The ICER for TMVr improved slightly to $£ 22241$ per QALY gained if we assumed that patients who underwent cardiac transplantation or LVAD placement at age $>65$ years would have died rather than receiving these treatments in the UK. If the cost of the TMVr device was reduced, TMVr cost-effectiveness improved (figure 4). At a device cost of $£ 13200$ (20\% less than the current cost), the ICER for TMVr was projected to be $<£ 20000$ per QALY. However, even if the TMVr device was offered for free, TMVr was not projected to reduce lifetime healthcare costs compared with GRMT alone. Finally, if costs unrelated to HF in future years were ignored, the ICER for TMVr decreased to $£ 15661$ per QALY, and the probability that TMVr would be cost-effective at a threshold of $£ 20000$ per QALY improved to 79\%.

Subgroup analyses are summarised in table 4. Results were generally consistent, with ICERs $<£ 30000$ per QALY across subgroups stratified by sex, MR severity, tricuspid regurgitation

B

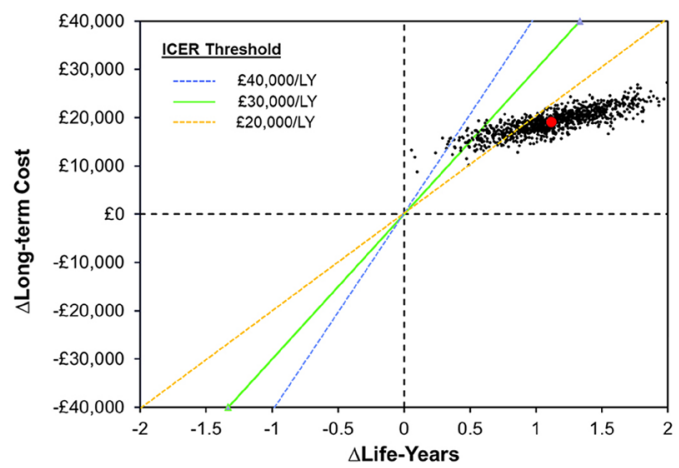

Figure 2 Joint distribution of lifetime incremental cost and quality-adjusted life-years for transcatheter mitral valve repair versus guidelinerecommended medical therapy. Incremental lifetime costs and benefits with TMVr versus GRMT are plotted on the cost-effectiveness plane with benefits expressed as quality-adjusted life-years (QALYs, A) and life-years (LYs, B). The solid red circle represents base-case estimates, the surrounding dots represent individual results for 1000 replicates of the study using bootstrap resampling, and the diagonal lines represent willingness-to-pay thresholds of $€ 30000$ (solid green), $£ 20000$ (dashed yellow) and $£ 40000$ (dashed blue) per QALY or LY gained. The base-case results demonstrated a gain of 0.82 QALYs and $1.17 \mathrm{LYs}$ at an incremental cost of $£ 19128$ per patient (after discounting), resulting in ICERs of $£ 23270$ per QALY (A) and f17140 per LY gained (B). Points above and to the left of the diagonal threshold lines represent ICERs greater than the threshold (unfavourable) and points below and to the right of the threshold lines represent ICERs less than the threshold (acceptable). 
Table 3 Projected lifetime costs, QALYs and incremental cost-effectiveness ratios under base-case assumptions and sensitivity analyses

\begin{tabular}{|c|c|c|c|c|c|c|c|c|c|}
\hline & \multicolumn{3}{|c|}{ Lifetime costs } & \multicolumn{3}{|c|}{ QALYs } & \multirow{2}{*}{$\begin{array}{l}\text { ICER } \\
\text { (£ per QALY) }\end{array}$} & \multirow{2}{*}{$\begin{array}{l}\text { Probability < } \\
\text { £20000 per } \\
\text { QALY }\end{array}$} & \multirow{2}{*}{$\begin{array}{l}\text { Probability < } \\
\text { £30000 per QALY }\end{array}$} \\
\hline & $\mathrm{TMVr}$ & GRMT & $\Delta$ & $\mathrm{TMVr}$ & GRMT & $\Delta$ & & & \\
\hline Base case & f51 029 & f31902 & f19128 & 3.42 & 2.6 & 0.82 & $£ 23270$ & $18 \%$ & $89 \%$ \\
\hline \multicolumn{10}{|l|}{ Discount rate } \\
\hline $0 \%$ & £59970 & f37990 & f21980 & 4.31 & 3.19 & 1.12 & £19607 & $57 \%$ & $96 \%$ \\
\hline $5 \%$ & $£ 47996$ & f29655 & f18311 & 3.11 & 2.38 & 0.73 & £25015 & $8 \%$ & $82 \%$ \\
\hline \multicolumn{10}{|l|}{ TMVr device cost } \\
\hline f0 & $£ 35294$ & f31902 & £3393 & 3.42 & 2.6 & 0.82 & $£ 4128$ & $100 \%$ & $100 \%$ \\
\hline f13200 (-20\%) & $£ 47882$ & f31 902 & f15981 & 3.42 & 2.6 & 0.82 & £19442 & $59 \%$ & $96 \%$ \\
\hline f19800 (+20\%) & f54176 & f31 902 & f22275 & 3.42 & 2.6 & 0.82 & £27099 & $3 \%$ & $70 \%$ \\
\hline \multicolumn{10}{|l|}{ Index procedure costs* } \\
\hline$\downarrow 50 \%$ & f49786 & f31902 & f17885 & 3.42 & 2.6 & 0.82 & f21758 & $32 \%$ & $93 \%$ \\
\hline$\uparrow 50 \%$ & f52272 & f31 902 & f20371 & 3.42 & 2.6 & 0.82 & £24782 & $9 \%$ & $83 \%$ \\
\hline \multicolumn{10}{|l|}{ Varying benefit of TMVr } \\
\hline 'Best case' scenariot & $£ 49881$ & f31902 & $£ 17979$ & 4.04 & 2.6 & 1.44 & f12494 & $99 \%$ & $100 \%$ \\
\hline 'Worst case' scenarioł & f51 240 & f31 902 & £19338 & 3.27 & 2.6 & 0.68 & $£ 28607$ & $2 \%$ & $60 \%$ \\
\hline Heart transplant/LVAD $=$ death & f50389 & f30862 & f19528 & 3.34 & 2.47 & 0.88 & $£ 22241$ & $26 \%$ & $92 \%$ \\
\hline $\begin{array}{l}\text { Excluding non-HF-related costs in years } \\
\text { of life added }\end{array}$ & £44 774 & f31 902 & f12873 & 3.42 & 2.6 & 0.82 & f15661 & $79 \%$ & $97 \%$ \\
\hline
\end{tabular}

*Excluding the cost of the TMVr device.

†Best case scenario: Survival benefit, health status benefit and cost benefit observed at 2 years remains constant throughout patient's lifetime.

$\neq$ Worst case scenario: No further survival benefit, health status benefit or cost benefit after 2 years (ie, $H R=1 ; \Delta \cos t=0 ; \Delta$ utilities $=0$ ).

GRMT, guideline-directed medical therapy; ICER, incremental cost-effectiveness ratio; QALY, quality adjusted life-year; TMVr, transcatheter mitral valve repair; $\Delta$, difference.

severity and New York Heart Association (NYHA) class. There was moderate heterogeneity in other subgroups, however, with ICERs $>£ 30000$ per QALY for patients aged $\geq 75$, patients with $\mathrm{LVEF} \geq 30 \%$, and those with ischaemic heart disease (with ICERs $<£ 20000$ per QALY for each of the complementary subgroups). Results were generally similar when cost-effectiveness was expressed in terms of cost per LYG (online supplemental tables C and D).

\section{DISCUSSION}

In this study using data from individual participants in the COAPT trial, we found that TMVr for HFrEF and severe secondary MR was reasonably cost-effective from a UK NHS perspective. In the 2 years after $\mathrm{TMVr}$, costs were reduced by

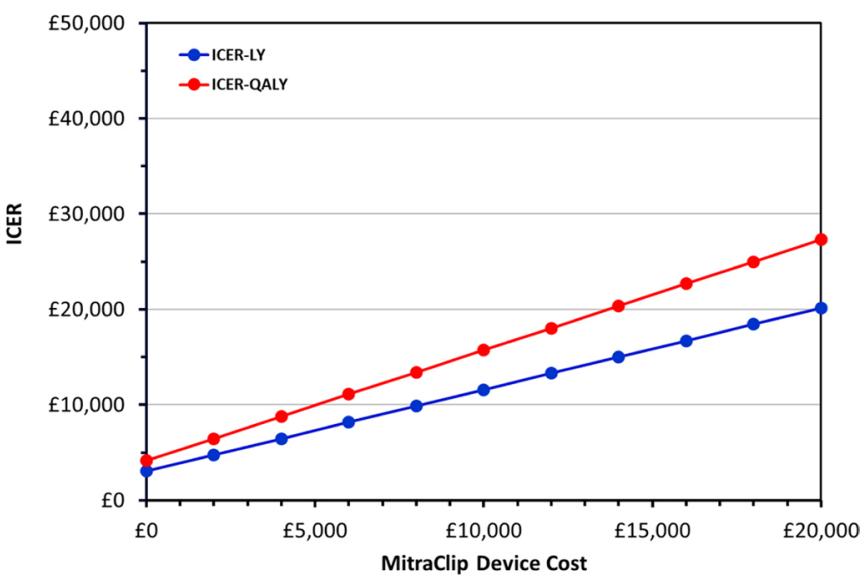

Figure 4 Sensitivity analysis - impact of alternative costs for the transcatheter edge-to-edge mitral valve repair (TMVr) device on the incremental cost-effectiveness ratio for TMVr compared with guidelinerecommended medical therapy (GRMT). Red line=ICER in cost per QALY gained; blue line=ICER in cost per LY gained. ICER, incremental costeffectiveness ratio. nearly $£ 4000$ compared with GRMT alone, partially offsetting the initial costs of TMVr. Nonetheless, total 2-year costs remained $\sim £ 14000 /$ patient higher with TMVr. When in-trial results were projected over a lifetime horizon, TMVr was associated with substantial gains in life expectancy and QALYs, resulting in life-time incremental cost-effectiveness ratios of $£ 17140$ per life-year gained and $£ 23270$ per QALY gainedvalues that are considered cost-effective by the National Institute for Health and Care Excellence (NICE) in the UK (ie, £20 $000-£ 30000$ per QALY gained). ${ }^{15}$

Two previous studies have examined the cost-effectiveness of TMVr from a UK perspective. An analysis using data from the EVEREST II trial found that the ICER for TMVr compared with GRMT for patients who were not candidates for mitral valve surgery was $\sim £ 13600$ per QALY gained. However, this analysis was based on patients with a mixture of primary and secondary MR and compared outcomes with historical controls. More recently, a disease-simulation model based on published aggregate data from the COAPT trial reported an ICER of $£ 30057$ per QALY ${ }^{16}$ - somewhat less favourable than our results despite assuming that the 2-year benefits of TMVr would persist indefinitely. The less-favourable ICER in this model-based analysis may reflect their markedly lower costs for GRMT (£10704) based on a 'typical' HFrEF population compared with our analysis (£31902), where costs were based on individual-patient data from the COAPT trial.

Our results should also be compared with economic evaluations of other cardiovascular therapies in the UK. Several studies have found that the ICER for transcatheter aortic valve intervention ranges from $£ 12000$ to $£ 14000$ per QALY gained for patients with severe, symptomatic aortic stenosis (AS) who are not candidates for surgical valve replacement. ${ }^{17}{ }^{18}$ However, these analyses excluded the costs of unrelated conditions, a common practice when disease-simulation models (rather than patient-level data) are used to evaluate cost-effectiveness. When these costs were excluded from our analysis, the ICER for TMVr was $\sim £ 16000$ per QALY. 
Table 4 Subgroup analyses (benefit in QALYs)

\begin{tabular}{|c|c|c|c|c|c|c|c|c|c|}
\hline & \multicolumn{3}{|c|}{ Lifetime costs (f) } & \multicolumn{3}{|l|}{ QALYs } & \multirow{2}{*}{$\begin{array}{l}\text { ICER } \\
\text { (fper QALY) }\end{array}$} & \multirow{2}{*}{$\begin{array}{l}\text { Probability < } £ 20000 \\
\text { per QALY }\end{array}$} & \multirow{2}{*}{$\begin{array}{l}\text { Probability < } \\
\text { £30000 per QALY }\end{array}$} \\
\hline & TMVr & GRMT & $\Delta$ & TMVr & GRMT & $\Delta$ & & & \\
\hline Base case & 51029 & 31902 & 19128 & 3.42 & 2.6 & 0.82 & 23270 & $18 \%$ & $89 \%$ \\
\hline \multicolumn{10}{|l|}{ Age } \\
\hline$<75(\mathrm{n}=323)$ & 66282 & 45729 & 20553 & 4.82 & 3.61 & 1.22 & 16916 & $80 \%$ & $97 \%$ \\
\hline$\geq 75(n=291)$ & 34804 & 15977 & 18827 & 1.93 & 1.43 & 0.5 & 38034 & $0 \%$ & $18 \%$ \\
\hline \multicolumn{10}{|l|}{ Sex } \\
\hline Male $(n=393)$ & 45915 & 26980 & 18935 & 2.95 & 2.2 & 0.76 & 25046 & $11 \%$ & $75 \%$ \\
\hline Female $(n=221)$ & 61341 & 39777 & 21564 & 4.36 & 3.23 & 1.13 & 19134 & $55 \%$ & $90 \%$ \\
\hline \multicolumn{10}{|l|}{ STS risk score } \\
\hline$<8(=352)$ & 62115 & 40875 & 21240 & 4.46 & 3.36 & 1.1 & 19309 & $59 \%$ & $96 \%$ \\
\hline$\geq 8(n=262)$ & 35679 & 20289 & 15390 & 1.98 & 1.61 & 0.37 & 41821 & $1 \%$ & $19 \%$ \\
\hline \multicolumn{10}{|l|}{ Aetiology of cardiomyopathy } \\
\hline Ischaemic $(n=373)$ & 43221 & 26635 & 16586 & 2.79 & 2.25 & 0.54 & 30715 & $3 \%$ & $49 \%$ \\
\hline Non-ischaemic $(n=241)$ & 63215 & 39994 & 23221 & 4.4 & 2.13 & 1.27 & 18270 & $65 \%$ & $94 \%$ \\
\hline \multicolumn{10}{|l|}{ Baseline LVEF } \\
\hline$<30 \%(n=274)$ & 55549 & 34046 & 21504 & 3.8 & 2.42 & 1.39 & 15482 & $91 \%$ & $100 \%$ \\
\hline$\geq 30 \%(n=301)$ & 47761 & 30143 & 17618 & 3.16 & 2.74 & 0.42 & 41650 & $3 \%$ & $24 \%$ \\
\hline \multicolumn{10}{|l|}{ Baseline mitral regurgitation } \\
\hline $3+(n=320)$ & 50443 & 32168 & 18275 & 3.47 & 2.75 & 0.72 & 25453 & $14 \%$ & $69 \%$ \\
\hline $4+(n=293)$ & 51651 & 31431 & 20220 & 3.38 & 2.38 & 1 & 20301 & $47 \%$ & $90 \%$ \\
\hline \multicolumn{10}{|l|}{ Baseline tricuspid regurgitation } \\
\hline Moderate or severe $(n=98)$ & 48181 & 28198 & 19982 & 3.41 & 1.73 & 1.68 & 11908 & $97 \%$ & $99 \%$ \\
\hline Mild or less ( $n=501)$ & 51444 & 32436 & 19008 & 3.41 & 2.73 & 0.68 & 28077 & $3 \%$ & $59 \%$ \\
\hline \multicolumn{10}{|l|}{ NYHA class } \\
\hline I or II $(n=240)$ & 55016 & 34940 & 20076 & 3.83 & 3.02 & 0.82 & 24603 & $23 \%$ & $68 \%$ \\
\hline III $(n=322)$ & 46750 & 30301 & 16449 & 3.07 & 2.42 & 0.65 & 25345 & $15 \%$ & $68 \%$ \\
\hline IV $(n=51)$ & 58186 & 29001 & 29185 & 3.32 & 2.04 & 1.28 & 22819 & $32 \%$ & $70 \%$ \\
\hline
\end{tabular}

GRMT, guideline-directed medical therapy; ICER, incremental cost-effectiveness ratio; LVEF, left ventricular ejection fraction; NYHA, New York Heart Association; QALY, quality adjusted life-year; $\mathrm{TMVr}$, transcatheter mitral valve repair; $\triangle$, difference.

Comparison of TMVr with other device-based therapies for patients with advanced heart failure is also instructive. Patientlevel analysis of the cost-effectiveness of cardiac resynchronisation therapy-pacemakers (CRT-P) versus GRMT for patients with NYHA class III/IV HFrEF found that life-time ICER for CRT was $\sim £ 6000$ per QALY gained. ${ }^{19}{ }^{20}$ When a CRT plus implantable cardioverter-defibrillator (CRT-ICD) device was compared with CRT-P, however, the lifetime ICER increased to $\sim £ 40000$ per QALY gained. Cost-effectiveness analyses of destination LVADs for end-stage HFrEF have reported ICERs consistently $>£ 50000$ per QALY gained. ${ }^{21} 22$

\section{Clinical and policy implications}

Our study provides several important insights that may inform future clinical and health policy decisions. In addition to the device cost, one of the most important factors in determining the cost-effectiveness of $\mathrm{TMVr}$ is the duration of benefit. In our base-case analysis, we assumed that the benefits of $\mathrm{TMVr}$ decrease gradually after 2 years of treatment; under these conditions, the ICER for TMVr versus GRMT was $\sim £ 23000$ per QALY gained. However, if the benefit of TMVr relative to GRMT persists long term, the ICER was much more favourable at $\sim £ 12000$ per QALY.

The sensitivity of our results to assumptions regarding durability of benefit emphasises the importance of competing risks in determining the cost-effectiveness of TMVr. For frail elderly patients, those with end-stage disease or life-threatening comorbidities, the cost-effectiveness of $\mathrm{TMVr}$ may be substantially less favourable than was observed in COAPT. Indeed, we identified several subgroups for which the ICER for TMVr exceeded
$£ 30000$ per QALY gained including patients aged $\geq 75$ or those with LVEF $\geq 30 \%$ for whom the gain in life expectancy compared with GRMT was small. For patients aged $\geq 75$, this reflected their poor prognosis despite intervention. For patients with LVEF $\geq 30 \%$, the small increase in life expectancy reflects their intrinsically better prognosis even in the absence of TMVr. Conversely, the ICERs for younger patients and those with LVEF $<30 \%$ were much more favourable. It is important to recognise that these subgroup analyses are relatively unstable, however, given their small sample sizes.

\section{Limitations}

Our study should be considered in light of several limitations. First, the COAPT trial was conducted entirely within the USA. As such, we used several approaches to adapt the results for the UK. With respect to outcomes, projections beyond the trial time horizon were based on UK-specific life-tables (calibrated to the trial population), and individual health utilities were derived from a UK-specific algorithm. ${ }^{12}$ Costs were assessed in a similar fashion by using NHS-specific tariffs to assign costs to all healthcare resources. Thus, the main assumption of the economic analysis is that the pattern of clinical outcomes (including HHF and mortality) would be similar between the UK and US healthcare systems - an assumption that has been used by many previous economic analyses. ${ }^{101123}$

Importantly, our lifetime projections of survival, QoL and costs beyond the trial period are uncertain and are unlikely to be validated by additional follow-up, because the study protocol allowed for cross-over to TMVr after 2 years. We therefore evaluated a range of alternative assumptions regarding the duration 
of benefits and found that, even under conservative assumptions, TMVr was cost-effective. We did not consider the Mitra-FR trial, since post-hoc analyses suggest that this trial may have enrolled rather different patients from COAPT. ${ }^{24}{ }^{25}$ We also did not consider mitral valve surgery as an alternative therapeutic option, since it is not recommended for secondary MR by current US or European guidelines. ${ }^{26} 27$

\section{CONCLUSIONS}

For patients similar to those enrolled in the COAPT trial, TMVr improves both life expectancy and QoL compared with GRMT alone and is cost-effective in the context of the UK NHS. Future research should focus on identifying patient subgroups who derive the greatest long-term benefit from TMVr in order to optimise the cost-effectiveness of this evolving therapy.

\section{Key messages}

\section{What is already known on this subject?}

- For patients with heart failure with reduced left ventricular ejection fraction (HFrEF) and severe secondary mitral regurgitation who remain symptomatic despite guidelinerecommended medical therapy (GRMT), edge-to-edge transcatheter mitral valve repair (TMVr) improves quality of life, and reduces both hospitalisations and death compared with GRMT alone.

- Previous studies have demonstrated that this therapy is reasonably cost-effective (but not cost saving) from the perspective of the US healthcare system, but the extent to which these findings can be extrapolated to other healthcare environments is unknown.

\section{What might this study add?}

- In this study, we found that TMVr using edge-to-edge repair was reasonably cost-effective from the perspective of the UK National Health Service.

- Although TMVr was not cost saving in either the short or long term, by improving survival and quality of life, TMVr provided good economic value compared with many other medical and procedural therapies.

\section{How might this impact on clinical practice?}

- These findings, which are derived from patient-level data from the COAPT (Cardiovascular Outcomes Assessment of the MitraClip Percutaneous Therapy for Heart Failure Patients with Functional Mitral Regurgitation) trial, the largest randomised trial of TMVr to date, suggest that this therapy should be funded by the National Health Service for appropriately selected patients (ie, those similar to the patients enrolled in COAPT).

\section{Author affiliations}

Clinical Trials Center, Cardiovascular Research Foundation, New York, New York, USA

${ }^{2}$ Department of Cardiology, St. Francis Hospital and Heart Center, Roslyn, New York, USA

${ }^{3}$ Cardiovascular Research, Saint Luke's Mid America Heart Institute, Kansas City, Missouri, USA

${ }^{4}$ Cardiology, Royal Brompton and Harefield NHS Foundation Trust, London, UK ${ }^{5}$ University of Glasgow Institute of Cardiovascular and Medical Sciences, Glasgow, UK

${ }^{6}$ Golden Jubilee National Hospital, Clydebank, UK

${ }^{7}$ Cardiology, University Hospital of South Manchester NHS Foundation Trust, Manchester, UK

${ }^{8}$ Division of Cardiovascular Medicine, The Ohio State University, Columbus, Ohio, USA
${ }^{9}$ Vanderbilt University Medical Center, Nashville, Tennessee, USA

${ }^{10}$ Baylor Scott \& White The Heart Hospital Plano, Plano, Texas, USA

${ }^{11}$ The Zena \& Michael A. Wiener Cardiovascular Institute, Icahn School of Medicine at Mount Sinai, New York, New York, USA

${ }^{12}$ Cardiovascular Research Foundation, New York, New York, USA

${ }^{13}$ University of Glasgow Robertson Centre for Biostatistics, Glasgow, UK

${ }^{14}$ National Heart \& Lung Institute, Royal Brompton \& Harefield Hospitals, Imperial College London, London, UK

Contributors Study concept and design: DJC, WA, JL, MJM, GWS, JGFC. Statistical analyses: DJC, KW, EM. Manuscript writing: DJC, JGFC. Critical revision of manuscript for content/interpretation: EM, RS, MCP, MHB, WA, JL, MJM, GWS. DJC accepts full responsibility for the conduct of the study, had access to the data. All authors controlled the decision to publish.

Funding The COAPT trial and the UK economic substudy were funded by Abbott (Santa Clara, California, USA). The funding agreement stipulated that the academic investigators had full access to the study data, performed all analyses, and had the right to publish the results regardless of the findings.

Competing interests DJC: Research grant support from Abbott, Edwards Lifesciences, Boston Scientific, Medtronic; consulting income from Abbott, Edwards Lifesciences, Boston Scientific, Medtronic. RS: Proctoring/Consultancy for Abbott. MCP: Research funding: Boehringer Ingelheim, Roche, SQ Innovations, AstraZeneca, Novartis, Novo Nordisk, Medtronic, Boston Scientific, Pharmacosmos. Consultancy and Clinical Trials Committees Committees: Boehringer Ingelheim, Novartis, AstraZeneca, Novo Nordisk, Medtronic, Abbvie, Bayer, Takeda, Cardiorentis, Pharmacosmos, Siemens. MCP is supported by the British Heart Foundation (BHF) Centre of Research Excellence Award (RE/13/5/30177 and RE/18/6/34217+). WA: Research grant support from Abbott Vascular; consulting income from Abbott Vascular. JL: Research grant support from AstraZeneca; consulting income from Abbott Vascular, CVRx, Edwards Lifesciences, RESMED, Relypsa, Boehringer Ingelheim and V-Wave. MJM: Served as co-primary investigator for the PARTNER Trial for Edwards Lifesciences and COAPT trial for Abbott; served as study chair for the APOLLO trial for Medtronic. GWS has received speaker or other honoraria from Terumo, Cook, Infraredx; has served as a consultant to Valfix, TherOx, Robocath, HeartFlow, Ablative Solutions, Vectorious, Miracor, Neovasc, Abiomed, Ancora, Elucid Bio, Occlutech, CorFlow, Reva, MAIA Pharmaceuticals, Vascular Dynamics, Shockwave, V-Wave, Cardiomech, Gore; and has equity/options from Ancora, Cagent, Applied Therapeutics, Biostar family of funds, SpectraWave, Orchestra Biomed, Aria, Cardiac Success, Valfix, MedFocus family of funds. JGFC: Research grants from Amgen, Bayer, Bristol Myers Squibb, Vifor, Pharacosmos, Cytokinetics, Johnson and Johnson, MyoKardia, Stealth Biopharmaceuticals and Viscardia; honoraria from Abbott, Bayer, Bristol Myers Squibb, Novartis, Medtronic, Idorsia, Vifor, Pharmacosmos, Cytokinetics, Servier, Boehringer-Ingelheim, AstraZeneca, Innolife, Torrent, Johnson \& Johnson, MyoKardia, Respicardia, Stealth Biopharmaceuticals, Viscardia. The other authors report no relevant disclosures.

\section{Patient consent for publication Not applicable.}

Ethics approval The COAPT trial was approved by relevant institutional review boards, and informed consent was obtained from all patients. The economic analysis was approved by the institutional review board at Saint Luke's Hospital of Kansas City. Participants gave informed consent to participate in the study before taking part.

Provenance and peer review Not commissioned; externally peer reviewed.

Data availability statement No data are available. By contractual arrangement with the study sponsor, patient-level data will not be shared.

Supplemental material This content has been supplied by the author(s). It has not been vetted by BMJ Publishing Group Limited (BMJ) and may not have been peer-reviewed. Any opinions or recommendations discussed are solely those of the author(s) and are not endorsed by BMJ. BMJ disclaims all liability and responsibility arising from any reliance placed on the content. Where the content includes any translated material, BMJ does not warrant the accuracy and reliability of the translations (including but not limited to local regulations, clinical guidelines, terminology, drug names and drug dosages), and is not responsible for any error and/or omissions arising from translation and adaptation or otherwise.

Open access This is an open access article distributed in accordance with the Creative Commons Attribution Non Commercial (CC BY-NC 4.0) license, which permits others to distribute, remix, adapt, build upon this work non-commercially, and license their derivative works on different terms, provided the original work is properly cited, appropriate credit is given, any changes made indicated, and the use is non-commercial. See: http://creativecommons.org/licenses/by-nc/4.0/.

\section{REFERENCES}

1 Stone GW, Lindenfeld J, Abraham WT, et al. Transcatheter mitral-valve repair in patients with heart failure. N Engl J Med 2018;379:2307-18. 
2 Arnold SV, Chinnakondepalli KM, Spertus JA, et al. Health Status After Transcatheter Mitral-Valve Repair in Heart Failure and Secondary Mitral Regurgitation: COAPT Trial. J Am Coll Cardiol 2019;73:2123-32.

3 Baron SJ, Wang K, Arnold SV, et al. Cost-effectiveness of transcatheter mitral valve repair versus medical therapy in patients with heart failure and secondary mitral regurgitation: results from the COAPT trial. Circulation 2019;140:1881-91.

4 Anderson JL, Heidenreich PA, Barnett PG, et al. ACC/AHA statement on cost/value methodology in clinical practice guidelines and performance measures: a report of the American College of Cardiology/American Heart Association Task Force on Performance Measures and Task Force on Practice Guidelines. J Am Coll Cardiol 2014;63:2304-22.

5 Husereau D, Drummond M, Petrou S, et al. Consolidated health economic evaluation reporting standards (CHEERS) statement. BMJ 2013;346:f1049.

6 NHS Improvement. National tariff payment system. Available: https://improvement. nhs.uk/resources/national-tariff/ [Accessed 12 Aug 2019].

7 British National Formulary. Available: https://bnf.nice.org.uk/drug/ [Accessed 9 Mar 2021].

8 NHS England. National schedule of reference costs. Available: https://www.england. nhs.uk/national-cost-collection/ [Accessed 11 Mar 2021]

9 Office for National Health Statistics, 2020. Available: https://www.ons.gov.uk/peop lepopulationandcommunity/birthsdeathsandmarriages/lifeexpectancies/datasets/nati onallifetablesunitedkingdomreferencetables [Accessed 1 Dec 2020].

10 Magnuson EA, Farkouh ME, Fuster V, et al. Cost-effectiveness of percutaneous coronary intervention with drug eluting stents versus bypass surgery for patients with diabetes mellitus and multivessel coronary artery disease: results from the FREEDOM trial. Circulation 2013;127:820-31.

11 Cohen DJ, Osnabrugge RL, Magnuson EA, et al. Cost-effectiveness of percutaneous coronary intervention with drug-eluting stents versus bypass surgery for patients with 3-vessel or left main coronary artery disease: final results from the synergy between percutaneous coronary intervention with Taxus and cardiac surgery (SYNTAX) trial. Circulation 2014;130:1146-57.

12 Brazier JE, Roberts J. The estimation of a preference-based measure of health from the SF-12. Med Care 2004;42:851-9.

13 Bang H. Estimating medical costs with censored data. Biometrika 2000:87:329-43.

14 Efron B. Better bootstrap confidence intervals. J Am Stat Assoc 1987;82:171-85.

15 National Institute for care and excellence, 2013. Available: https://www.nice.org.uk/ process/pmg9/chapter/the-reference-case [Accessed 1 Dec 2020].
16 Shore J, Russell J, Frankenstein L, et al. An analysis of the cost-effectiveness of transcatheter mitral valve repair for people with secondary mitral valve regurgitation in the UK. J Med Econ 2020;23:1425-34.

17 Orlando R, Pennant M, Rooney S, et al. Cost-effectiveness of transcatheter aortic valve implantation (TAVI) for aortic stenosis in patients who are high risk or contraindicated for surgery: a model-based economic evaluation. Health Technol Assess 2013;17:1-86.

18 Brecker S, Mealing S, Padhiar A, et al. Cost-utility of transcatheter aortic valve implantation for inoperable patients with severe aortic stenosis treated by medical management: a UK cost-utility analysis based on patient-level data from the ADVANCE study. Open Heart 2014;1:e000155.

19 Calvert MJ, Freemantle N, Yao G, et al. Cost-effectiveness of cardiac resynchronization therapy: results from the CARE-HF trial. Eur Heart J 2005;26:2681-8.

20 Yao G, Freemantle N, Calvert MJ, et al. The long-term cost-effectiveness of cardiac resynchronization therapy with or without an implantable cardioverter-defibrillator. Eur Heart J 2007;28:42-51.

21 Clegg AJ, Scott DA, Loveman E, et al. The clinical and cost-effectiveness of left ventricular assist devices for end-stage heart failure: a systematic review and economic evaluation. Health Technol Assess 2005;9:1-132.

22 Clarke A, Pulikottil-Jacob R, Connock M, et al. Cost-effectiveness of left ventricular assist devices (LVADs) for patients with advanced heart failure: analysis of the British NHS bridge to transplant (BTT) program. Int J Cardiol 2014;171:338-45.

23 Magnuson EA, Li H, Wang K, et al. Cost-Effectiveness of Long-Term Ticagrelor in Patients With Prior Myocardial Infarction: Results From the PEGASUS-TIMI 54 Trial. $J$ Am Coll Cardiol 2017;70:527-38.

24 Obadia J-F, Messika-Zeitoun D, Leurent G, et al. Percutaneous repair or medical treatment for secondary mitral regurgitation. N Engl J Med 2018;379:2297-306.

25 Grayburn PA, Sannino A, Packer M. Proportionate and Disproportionate Functional Mitral Regurgitation: A New Conceptual Framework That Reconciles the Results of the MITRA-FR and COAPT Trials. JACC Cardiovasc Imaging 2019;12:353-62.

26 Nishimura RA, Otto CM, Bonow RO, et al. 2017 AHA/ACC focused update of the $2014 \mathrm{AHA} / \mathrm{ACC}$ guideline for the management of patients with valvular heart disease: a report of the American College of Cardiology/American heart association Task force on clinical practice guidelines. Circulation 2017; 135:e1159-95

27 Baumgartner H, Falk V, Bax JJ, et al. 2017 ESC/EACTS guidelines for the management of valvular heart disease. Eur Heart J 2017;38:2739-91. 